\title{
Whom should we divert and to where? Looking at arrests in a I0-year cohort study of persons with severe mental illness William Fisher
}

\author{
Address: University of Massachusetts Medical School, 55 Lake Avenue North, WSH, 8B-23, Worcester, MA 01655, USA \\ from WPA Thematic Conference. Coercive Treatment in Psychiatry: A Comprehensive Review \\ Dresden, Germany. 6-8 June 2007 \\ Published: 19 December 2007 \\ BMC Psychiatry 2007, 7(Suppl I):S38 doi:10.1 I86/I47I-244X-7-SI-S38
}

This abstract is available from: http://www.biomedcentral.com/I47I-244X/7/SI/S38

(c) 2007 Fisher; licensee BioMed Central Ltd.

\section{Background}

For the last decade, diverting arrestees with mental illness from the criminal justice to the mental health systems has been a mainstay of US mental health policy. However, data allowing us to gauge the size and charge characteristics of arrestees with mental illness have been generally unavailable to planners and policymakers in this area. This paper represents an effort toward providing such data.

\section{Methods}

We examined charges lodged against a cohort of nearly 14,000 adults with severe mental illness receiving mental health services in Massachusetts in 1992 and followed for roughly 10 years, and also examined the relationship between services received, individual characteristics, and likelihood of offending.

\section{Results}

Roughly $30 \%$ of all cohort members were arrested at least once, many for serious felony charges. Moreover, our findings suggest that: (a) substantial numbers of arrestees with mental illness do not fit the profile of the stereotypical "low-level misdemean and diversion client"; (b) that services such as residential programing and case management are effective, but not across the full spectrum of arrestees, and (c) that correlates of poor performance in such settings include factors known to increase risk for offending in the general population.

\section{Conclusion}

These findings suggest that "criminogenic" risk factors be considered alongside mental health issues in planning the next generation of policy and diversion services for addressing this problem. 